\title{
Gestión ambiental de las empresas públicas y privadas en la ciudad de Guayaquil - Ecuador y su incidencia en el desarrollo sostenible ${ }^{*}$
}

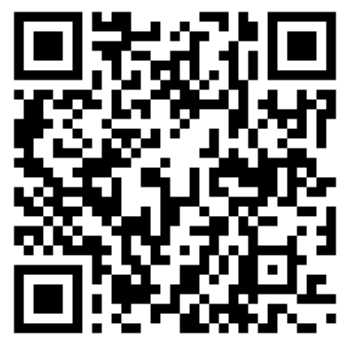

Environmental management of public and private companies in the city of Guayaquil - Ecuador and its impact on sustainable development

Elizabeth Eleana Malavé González ${ }^{* *}$ Mario Alfredo Fernández Ronquillo***

\section{Resumen}

La presente investigación trata sobre la Gestión ambiental de las empresas públicas y privadas y su incidencia en el desarrollo sostenible, el objetivo es poder determinar la gestión ambiental realizada por diferentes empresas públicas y empresas privadas para conocer su incidencia en el desarrollo sostenible. El diseño de investigación fue de tipo cualitativo con un enfoque exploratorio, la muestra seleccionada fue a convenir. La recolección de datos se realizó a través de un cuestionario de preguntas abiertas y de escala. Los resultados de la investigación revelaron que la gestión ambiental de las empresas públicas y privadas no son determinantes para el desarrollo sostenible, porque a pesar de que todas las empresas entrevistadas conocen sobre las regulaciones vigentes y las BPA

\footnotetext{
* Artículo original derivado del Proyecto "El desarrollo sostenible desde el análisis de la académia" Financiado por Universidad Estatal de Milagro entre agosto 2017 y mayo 2019

${ }^{* *}$ Docente universitario, Instituto Nacional de Pesca, Universidad Estatal de Milagro. Milagro, Ecuador. E-mail: lielymag@hotmail.com. ORCID: http://orcid.org/0000-0002-0188-0981.

*** Docente universitario, Instituto Nacional de Pesca, Universidad Estatal de Milagro. Milagro, Ecuador. E-mail: mfernandezr@unemi.edu.ec. ORCID: http://orcid.org/0000-0002-3630-102X.
}

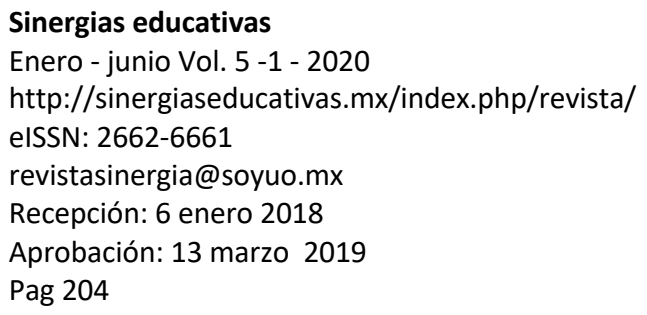

Disponible en

http://www.redalyc.org/articulo.oa?id=57356143200 15

Atribución/Reconocimiento-NoComercialCompartirlgual 4.0 Licencia Pública Internacional CC BY-NC-SA 4.0 https://creativecommons.org/licenses/by-ncsa/4.0/legalcode.es 
emitidas por el MAE, solo se aplica el 50\% de ellas como son: de Energía, agua, desechos, papel y responsable consumo; además poseen el $70 \%$ de personal capacitado en temas ambientales. Concluyendo, que es necesario incentivar el total cumplimiento de las BPA emitidas por el MAE y la incorporación de personal experto en temas ambientales a las organizaciones con la finalidad de crear motivación en los trabajadores para que adopten un comportamiento que involucre acciones de responsabilidad social y compromiso con el ambiente.

Palabras clave: gestión ambiental, empresas públicas, empresas privadas, desarrollo sostenible.

\begin{abstract}
This research is about the environmental management of public and private companies and their impact on sustainable development, the objective is to determine the environmental management carried out by different public companies and private companies to know their impact on sustainable development. The research design was quantitative with an exploratory approach, the selected sample was to be agreed. Data collection was carried out through a questionnaire with open and scaled questions. The results of the investigation revealed that the environmental management of public and private companies are not determinants for sustainable development, because although all the companies interviewed know about the current regulations and the BPAs issued by the MAE, only the $50 \%$ of them such as: Energy, water, waste, paper and responsible consumption and have $70 \%$ of personnel trained in environmental issues. In conclusion, it is necessary to encourage full compliance with the BPAs issued by the MAE and the incorporation of experts in environmental matters to organizations in order to create motivation for workers to adopt a behavior that involves actions of social responsibility and commitment with the environment.
\end{abstract}

Key words: environmental management, public enterprises, private businesses, sustainable development. 


\section{Introducción}

Se define a la gestión ambiental de las empresas como el conjunto de actividades, mecanismos, acciones e instrumentos, dirigidos a garantizar la administración y uso racional de los recursos naturales, a través, de la conservación, mejoramiento, rehabilitación, monitoreo del medio ambiente y control de las actividades realizadas por el ser humano. La gestión ambiental requiere la aplicación de la política ambiental, considerando la cultura de la sociedad, la experiencia acumulada y la participación ciudadana (RodríguezCórdova, 2016, p. 7).

Las empresas deben comprometerse de manera periódica a analizar nuevas tecnologías o procesos que permitan minimizar los impactos. El sistema de gestión medio ambiental tiene el compromiso de cumplir con la normativa legal vigente, y toda organización puede establecer sus propias políticas ambientales internas para ser implantadas de acuerdo a la razón de ser de las mismas, apegándose siempre a la Guía de Buenas Prácticas Ambientales emitida por el Ministerio del Ambiente.

\section{Gestión ambiental en el sector público}

Es necesario que en la gestión ambiental se involucren representantes para la toma de decisiones, además de considerar los principios explicados anteriormente. Asimismo, se deben unir esfuerzos para emprender y coordinar las acciones con los sectores económicos y sociales del país. Brindar la información es de vital importancia para garantizar el cumplimiento de las políticas ambientales.

Para garantizar la aplicación de cualquier sistema de gestión ambiental se deben establecer alianzas entre las instituciones públicas y privadas y la comunidad involucrada, lo que permitirá diseñar políticas orientadas a los problemas y necesidades de cada sector para aplicar un modelo de desarrollo sostenible.

El estado debe asumir la responsabilidad de formar el talento humano con la finalidad de lograr un desarrollo sostenible mediante la dirección de funcionarios encargados de la gestión ambiental. Por 
esta razón, es importante que se agreguen temas relacionados al medio ambiente en los programas curriculares de las diferentes disciplinas (Hernández et al., 2015).

\section{Punto Verde}

El Ministerio de Ambiente de la República del Ecuador (MAE), con el fin de estimular al sector gubernamental y privado a la aplicación de novedosas y relevantes prácticas, ha desarrollado la herramienta Punto Verde para impulsar que el sector industrial y de servicios se vuelva competitivo en busca de la protección y preservación del ambiente.

Punto Verde se consigue por medio de un proceso de "Reconocimiento Ecuatoriano Ambiental" REA que se encuentra establecido en el (Acuerdo Ministerial 131, 2010), que será otorgado a las instituciones que logren reducir su porcentaje de contaminación mediante la implementación de Buenas Prácticas Ambientales. La evaluación se realizará a través de la confrontación de indicadores de por lo menos dos años de actividades ejecutadas en relación a los desechos, papel, uso del agua, energía y combustibles; capacitación $\mathrm{y}$ adquisiciones responsables.

\section{Desarrollo sostenible}

En la década de los años sesenta debido al agotamiento de los recursos no renovables y del deterioro creciente del medio natural ocasionado por los desechos de la civilización industrial se proliferaron graves problemas ambientales producidos en países de la OCDE (Organización para la Cooperación y el Desarrollo Económicos), por lo tanto, se empieza a tomar conciencia de las necesidades del medio ambiente. Posteriormente, se pudo evidenciar la acumulación de las emisiones, cambio climático, contaminación del océano, erosión de la capa de ozono, destrucción de la masa forestal, etc.

Como consecuencia, de la creciente conciencia ambiental se redactaron documentos en los cuales se estableció la importancia del ambiente, y dio origen a la celebración de varias conferencias ambientales alrededor del mundo por parte de la Organización de las 
Naciones Unidas. Las conferencias más importantes se celebraron en Estocolmo, Rio de Janeiro y Johannesburgo en los años de 1972, 1992 y 2002 respectivamente (Gómez, 2014, p. 10).

\section{Dimensiones del desarrollo sostenible}

Es importante el fortalecimiento del ámbito institucional para alcanzar el desarrollo sostenible con la finalidad de que responda de forma coherente y eficaz a los desafíos actuales y del futuro para reducir los vacíos en la ejecución de la agenda de desarrollo sostenible. Por lo tanto, se reafirma que es necesario reforzar la gestión del Estado en términos ambientales internacionales con la finalidad de fomentar la integración del desarrollo sostenible mediante las dimensiones económica, social y ambiental; y a su vez, la coordinación en el sistema de las Naciones Unidas.

\section{Dimensión económica}

La economía es la ciencia que estudia la forma en que se utilizan los recursos escasos para satisfacer necesidades ilimitadas con la finalidad de producir bienes y servicios para la sociedad. Un sistema económico es aquel que se refiere al conjunto de relaciones que caracterizan la organización económica de la sociedad y la actividad económica corresponde a la acción relacionada a la producción, distribución y consumo de bienes y servicios (Riestra, 2018, p. 26).

La dimensión económica es esencial para el desarrollo sostenible, considerando que el mercado puede aprovechar la aplicación de regulaciones ambientales nacionales e internacionales, la utilización de procesos de producción más limpia y eficiente con agregación de valor en las materias primas; haciendo necesaria la racionalidad en la utilización de los recursos (Riestra, 2018, p. 27).

Se refiere a que la producción, y el intercambio de bienes y servicios se deben ajustar en beneficio del desarrollo sustentable. De la misma manera, se obliga a dar cumplimiento a las diferentes regulaciones nacionales e internacionales, realizar procesos de producción más limpios y eficientes para la agregación de valor a las materias primas que hayan utilizado estos procesos. Es decir, se emplean los elementos y procesos fundamentales de la economía, pero se incluye 
la sostenibilidad de acuerdo a la racionalidad ambiental. La Dimensión Económica del Desarrollo Sustentable, utiliza elementos esenciales de la economía y sus procesos, pero también incluye un componente de sustentabilidad, fundamentado en la racionalidad ambiental (Riestra, 2018, p.27).

\section{Dimensión social}

El desarrollo sostenible también se encuentra orientado a mejorar la calidad de vida de la sociedad, mediante la utilización de componentes sociales y culturales que influyen en el desarrollo de los pueblos. Busca satisfacer las necesidades básicas de todos los seres humanos y poner fin a la pobreza mediante la implementación de un plan de educación en todos los niveles, o una educación integral que permita la formación de las personas de acuerdo a los requerimientos de la sociedad y de las capacidades de las personas, sin desatender los valores éticos con la finalidad de lograr el desarrollo de la sociedad (Riestra, 2018, p.27).

\section{Dimensión ambiental}

Los recursos naturales son utilizados en la producción de bienes y servicios para satisfacer las necesidades de las personas, pero no es posible contemplar un desarrollo o concebir la vida humana sin los recursos de la naturaleza. Por cuanto, se debe tener racionalidad para aprovechar y utilizar el capital natural (Riestra, 2018, p.28).

El desarrollo sostenible incorpora la dimensión ambiental que es fundamental para la preservación del medio ambiente mediante el aprovechamiento racional de los recursos para garantizar la sostenibilidad del desarrollo, se limita la utilización de los recursos naturales de acuerdo a la capacidad que tiene el medio natural para recuperarse o regenerarse. En los procesos productivos se puede observar que la generación de los residuos se limita a la capacidad de asimilación del ecosistema (Riestra, 2018, p.28).

\section{Cultura y educación ambiental}

Aunque se han realizado trabajos de preservación no se ha logrado reducir la degradación ambiental, es por esto que este tema ha 
conseguido mayor importancia para estudiosos y ciudadanos. Para obtener conciencia ambiental, es necesario utilizar los medios informativos del conocimiento ambiental que permita alcanzar el desarrollo sostenible mediante nuevas estrategias. Lo que genera mayor impacto es el fortalecimiento de la educación y mejoramiento de la conciencia de los individuos con la finalidad de entender la dimensión de los problemas, tratando en lo posible de impulsar el respeto a la vida (Severiche, 2016, p.268).

La cultura requiere que los individuos y grupos participen activamente para que tengan una perspectiva convergente a favor de la naturaleza. Por lo tanto, la cultura es parte de la vida y armonía de la población. Las creencias, hábitos, usos, costumbres y tradiciones corresponden a diferentes culturas; por esto, nuestros ancestros tenían mayor conciencia y cuidado con el ambiente. Actualmente, se ha rebasado la capacidad de convivencia armónica con la naturaleza fomentado por el consumo y la política del mínimo esfuerzo impulsada por el modelo de desarrollo económico (Severiche et al., 2016).

De manera contundente, se puede decir que cualquier país debe utilizar la educación ambiental con el propósito de abordar e intervenir dicha problemática, considerando la multiculturalidad de los países que los identifica como tesoro patrimonial.

\section{Cumbre para el Desarrollo Sostenible}

Durante la Cumbre sobre el Desarrollo Sostenible realizada en septiembre de 2015, los estados miembros de la Organización de las Naciones Unidas (ONU) aprobaron la Agenda 2030 que incluye 17 Objetivos de Desarrollo Sostenible para erradicar la pobreza, la desigualdad, la injusticia, enfrentar el cambio climático fundamentados en los logros alcanzados por los Objetivos de Desarrollo del Milenio (ODM) que abordan causas como la pobreza y desarrollo para todas las personas. Cada objetivo posee metas específicas que se deben alcanzar en los próximos 11 años, para lograrlo los gobiernos, el sector privado, la sociedad civil y todas las personas en general tienen que hacer su parte. 
La Organización de las Naciones Unidas ONU publicó que los Jefes de Estado y de Gobierno y los Altos Representantes resolvieron hasta el 2030; velar por el cuidado del planeta y de los recursos naturales. Adicionalmente, se establece crear circunstancias para un crecimiento económico sostenible, que permita que todos tengan trabajo decente, considerando los distintos grados de desarrollo y capacidades individuales de cada nación.

\section{Desarrollo sostenible empresarial}

Desde el punto de vista organizacional, el desarrollo sostenible se encuentra basado en la ecoeficiencia, es decir, producir más con menor utilización de recursos y menor contaminación. El término ecoeficiencia resultó en la Cumbre de la Tierra de las Naciones Unidas en Río de Janeiro en 1992 cuando el Consejo para el Desarrollo Sostenible, World Business Council for Sustentainable Development, fundado por Stephan Schmidheiny, publicó el libro denominado "Cambiando el Curso" Changing Course, en el cual se realiza un análisis de la compatibilidad existente del crecimiento económico con la protección medio ambiental y se proponen soluciones para transformar los costos en el tratamiento de los recursos naturales, incitando a las empresas a ser más competitivas, más innovadoras y más responsables con el entorno (Isaac et al., 2017, p.28).

El World Business Council for Sustentainable Development WBCSD establece el siguiente concepto de ecoeficiencia:

La ecoeficiencia se obtiene a través del suministro de bienes y servicios a precios competitivos, que satisfagan las necesidades humanas proporcionando calidad de vida, y a su vez reduciendo progresivamente los impactos ecológicos y consumo de recursos a lo largo de su ciclo de vida, hasta un nivel que se encuentre acorde con la capacidad de carga estimada de la Tierra.

Como resultado de la utilización de este nuevo marco competitivo, las grandes empresas no sólo implementan sistemas de gestión ambiental, mejoran tecnologías o desarrollan productos amigables con el ambiente, sino que comienzan a recomendar a sus proveedores o industrias auxiliares, por lo general medianas empresas, que sigan 
los mismos pasos ambientales con la finalidad de diseñar a largo plazo una amplia estrategia de garantías competitivas que garanticen la supervivencia del grupo industrial y a su vez los beneficios de aplicarlas (Climent y Giner, 2001, p. 125).

\section{Sustentabilidad}

En lo sustentable intervienen valores que deberían ser específicos en el comportamiento humano, el comprender que los recursos de una sociedad son escasos ante unas necesidades humanas diversas e ilimitadas y la relación existente con los límites de crecimiento económico, la necesidad de realizar la transformación del sistema económico dominante con la finalidad de garantizar que la industria y la agricultura generen energías limpias utilizando recursos renovables, la satisfacción de las necesidades de las presentes generaciones sin comprometer las futuras, con la finalidad de encontrar el bien común. Estos fundamentos de lo sustentable crean un nuevo enfoque sobre la necesidad de cambiar la mentalidad humana, mediante una revolución cultural en la educación y en los valores sociales (Zarta, 2018, p. 412).

El desarrollo económico sostenible se puede alcanzar cuando las empresas o personas adoptan un criterio rentable, hacen que sus proyectos sean financieramente exitosos, sin afectar que al mismo tiempo abran su capital financiero a más socios, tengan un trato digno hacia sus trabajadores y clientes, cancelando buenos salarios a la comunidad. La sostenibilidad social se consigue cuando se apoyan proyectos en el mantenimiento de la relación comunitaria para emprender objetivos comunes que permitan mejorar las condiciones de vida, como por ejemplo las ONG sociales y ambientalistas. La sostenibilidad ambiental se podrá obtener siempre y cuando la explotación de los recursos naturales permanezca dentro de los límites de la regeneración y el crecimiento natural, empezando con planear la explotación de los recursos y precisar los efectos que tendrá la misma en el ecosistema (Zarta et al., 2018). En la Comisión Brundtland en el año 1991, se reconoció que el concepto de sustentabilidad debe ir más allá del aspecto ambiental, considerando el bienestar humano, ecológico y sus interacciones. (Carro, Sarmiento, y Rosano, 2017, p. 355). 


\section{La sustentabilidad organizacional}

El concepto de sustentabilidad comenzó a utilizarse durante la década del 80 a través de la expresión de desarrollo sustentable. Este desarrollo permite alcanzar un mayor bienestar económico sin descuidar los efectos ocasionados al medio ambiente y a la sociedad. Esto implica el equilibrio entre las dimensiones económica, social y ambiental. Es decir, satisfacer las necesidades de las presentes generaciones sin comprometer la capacidad de las futuras generaciones de satisfacer las suyas (Blázquez y Peretti, 2012, p. 41).

Una forma de operar en este sentido es conjuntamente con la responsabilidad social empresarial. La RSE es una autorregulación que se encuentra incorporada a la gestión del negocio que incluye los aspectos sociales y ambientales. Esta autorregulación incorpora leyes locales, acuerdos, tratados globales y normas internacionales, con la finalidad de satisfacer las necesidades de los interesados (Blázquez y Peretti, 2012, p. 42). Varios organismos internacionales como la Comisión Económica para América Latina y el Caribe (CEPAL), el Instituto Ethos de Responsabilidad Social, el European Bahaí Business Forum, el Business in the Community, la International Organization for Stan-dardization (ISO) han desarrollado propuestas para orientar, fomentar, regular y promover la RSE. Por esta razón, la ISO 26000 establece una guía con la finalidad de apoyar a las empresas a demostrar su compromiso de forma transparente con los grupos de interés y a su vez incrementar la credibilidad de sus informes (López, Ojeda, y Ríos, 2017, p.37).

\section{La sustentabilidad desde la perspectiva de la misión y la visión}

La visión está relacionada al conjunto de ideales, valores, sueños y creencias que identifica a las organizaciones y las orienta hacia el futuro. Es decir, se refiere a la búsqueda de un futuro determinado para la organización en concordancia con los ideales y principios de su razón de ser; mientras que la misión es el objetivo que no cuenta con un plazo definido sin límite de tiempo para alcanzarlo (Blázquez et al., 2012).

Se puede decir con certeza que los conceptos de visión y misión representan la forma concreta en que se operacionaliza la 
sustentabilidad de la empresa. Es decir, se podrán modificar en el tiempo como resultados de las realidades y procesos de adaptación, debido a que la finalidad de toda organización es convertirse en sustentable. Desde este punto de vista, la sustentabilidad se aplica transversalmente en el conjunto de organizaciones, que facilitan los procesos de adaptación y cambio.

Actualmente, la visión y la misión se deben integrar en la sustentabilidad, considerando un cambio en el paradigma de las mismas que permita incorporar términos de sustentabilidad a través de la rentabilidad, hacia lo interior y exterior de la organización mediante la adecuación del concepto de sustentabilidad dentro de la visión y misión organizacional.

\section{Incentivos para la responsabilidad social}

Las organizaciones poseen diferentes incentivos para involucrarse en gestiones de responsabilidad social, el compromiso que adopten dependerá de la visión de cada empresario y los resultados que de ella se espera obtener. Es decir, las empresas se hacen responsables socialmente cuando las ganancias involucran a la ley como un requisito principal que se debe cumplir. En la segunda, el empleador trata de satisfacer las expectativas de la comunidad, en temas del medio ambiente (Hernandez, Mendoza, y Salazar, 2017, p. 176).

La responsabilidad social corporativa nace como respuesta de las grandes corporaciones $\mathrm{y}$ organismos internacionales para contrarrestar la imagen negativa ocasionada por el sistema capitalista neoliberal, y mantenerse en el mercado nacional e internacional a largo plazo, mediante la incorporación de principios éticos y una visión global flexible al cambio del entorno. Buscan minimizar y suavizar los efectos negativos provocados al ambiente y a la sociedad, la empresa privada ahora cuenta con la responsabilidad social, el mercado, la competencia y la maximización de las ganancias, como elementos fundamentales del desarrollo sustentable (Chirinos et al., 2012). 


\section{Materiales y métodos}

El diseño de investigación fue de carácter exploratorio, con un muestreo por conveniencia. La recolección de datos se realizó a través de un cuestionario de preguntas abiertas y de escala, las cuales se encontraban orientadas a determinar la gestión ambiental realizada por diferentes empresas públicas y empresas privadas para conocer su incidencia en el desarrollo sostenible.

La muestra fue tomada mediante una técnica no probabilística y no aleatoria debido a que se han tomado en consideración individuos que faciliten el acceso a la información y a su vez, la disponibilidad de las personas que forman parte de la muestra.

El levantamiento de la información se realizó a aquellas empresas públicas y empresas privadas establecidas en el cantón Guayaquil. Se consideró 5 empresas públicas, como: Dirección Provincial del Consejo de la Judicatura - Guayas, Instituto Antártico Ecuatoriano, Instituto Nacional de Pesca, SENESCYT y Secretaría Técnica de Planificación (Zona 8); y 5 empresas privadas como: Banco del Litoral, Banco Pichincha, Corporación La Favorita, GEA Ecuador, Innova-MKT S.A.

\section{Resultados}

El cuestionario fue aplicado en el mes de junio de 2019. En donde se consideró una muestra de $50 \%$ de empresas públicas y $50 \%$ de empresas privadas. Con respecto al conocimiento de las regulaciones y Buenas Prácticas Ambientales obligatorias que por ley se deben utilizar, se determina que todas las empresas públicas y empresas privadas entrevistadas conocen sobre las regulaciones vigentes, y las BPA emitidas por el Ministerio del Ambiente.

En la Figura 1, se puede observar el porcentaje de regulaciones ambientales obligatorias aplicadas por empresas públicas y empresas privadas, en la cual se determina que el $30 \%$ de las empresas entrevistadas se encuentran aplicando las regulaciones ambientales entre los rangos $(30 \%-40 \%)$ y $(90 \%-100 \%)$, el $20 \%$ entre los rangos de $(70 \%-80 \%)$ y el $10 \%$ entre los rangos de $(10 \%-20 \%)$ y $(50 \%-60 \%)$. 


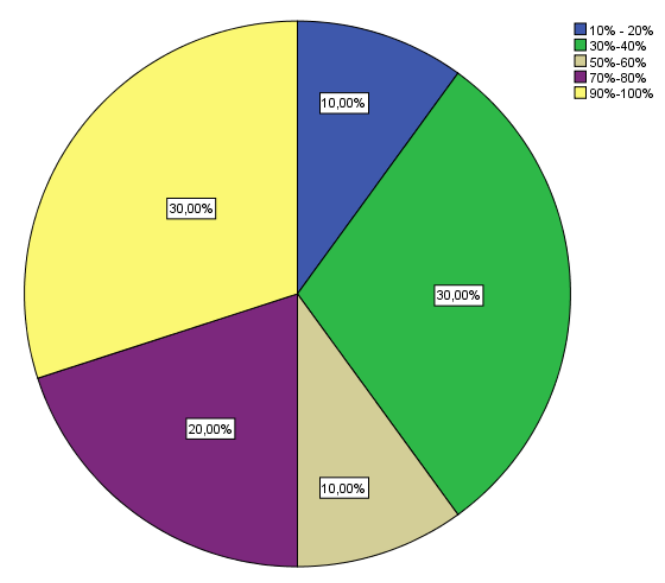

\section{Figura 1.}

Porcentaje de regulaciones ambientales obligatorias aplicadas por empresas públicas y empresas privadas

Elaboración propia

Referente a la implementación de políticas ambientales internas en las empresas públicas y empresas privadas, el $80 \%$ de las empresas si han implementado políticas ambientales internas y el $20 \%$ no lo ha hecho. Respecto a las Buenas Prácticas Ambientales que aplican las empresas públicas y empresas privadas, se determina que existe un mayor número de empresas que aplican las BPA referentes a la "Energía, agua, desechos, papel y responsable consumo", en un 50\%; el 20\% "desechos" y "papel"; y tan sólo el 10\% se dedican a aplicar el "responsable consumo". Como se observa en la Figura 2.

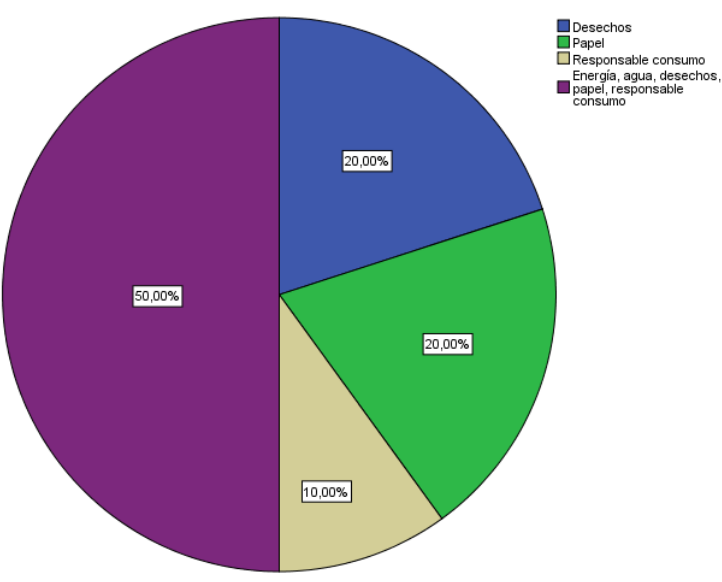




\section{Figura 2}

Buenas Prácticas Ambientales aplicadas por las empresas públicas y empresas privadas

Elaboración propia

Se ha identificado que sólo el $70 \%$ de las empresas entrevistadas tienen incorporado en su nómina de talento humano personal capacitado en temas ambientales y el $30 \%$ no, pero reciben comunicados vía correo electrónico que deben ser replicados para los demás compañeros de la organización. Como se puede observar en la Figura 3.

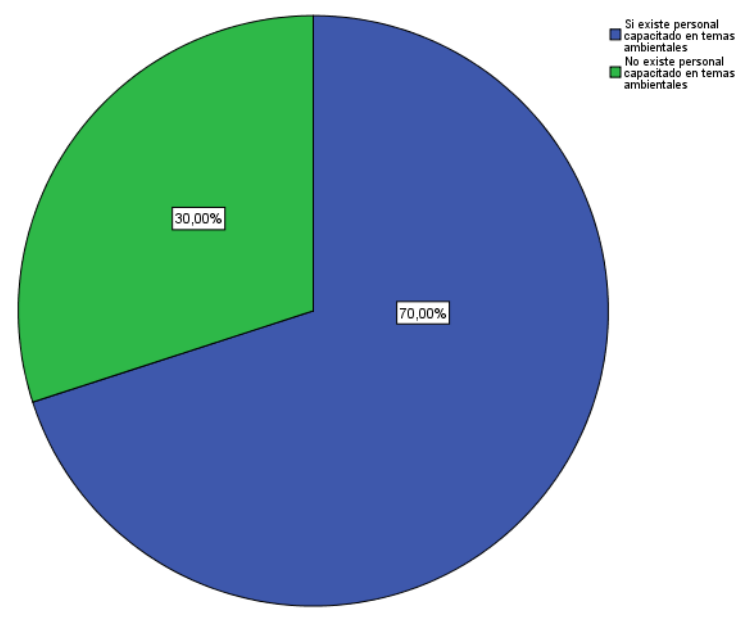

\section{Figura 3}

Existe personal capacitado en temas ambientales

Elaboración propia

En relación a la preocupación ambiental que tienen los trabajadores de las empresas públicas y empresas privadas por la conservación y mejora del medio ambiente, los resultados indican que el $40 \%$ de las empresas se sienten "muy despreocupados" debido a que las personas no se preocupan del daño ambiental que puedan causar y, sólo buscan su comodidad, lo que se refleja en las actitudes y preferencias de los individuos, mientras que el 30\% se encuentran "muy preocupados" buscando fomentar en sus colaboradores la conciencia ambiental para que aparte de ser aplicada en la organización también la lleven a sus hogares. Adicionalmente, se evidencia que el $30 \%$ están "relativamente preocupados", lo cual 
genera una inquietud porque de manera general los trabajadores no tienen preocupación alguna por la conservación y mejora del medio ambiente. Como se muestra en la Figura 4.

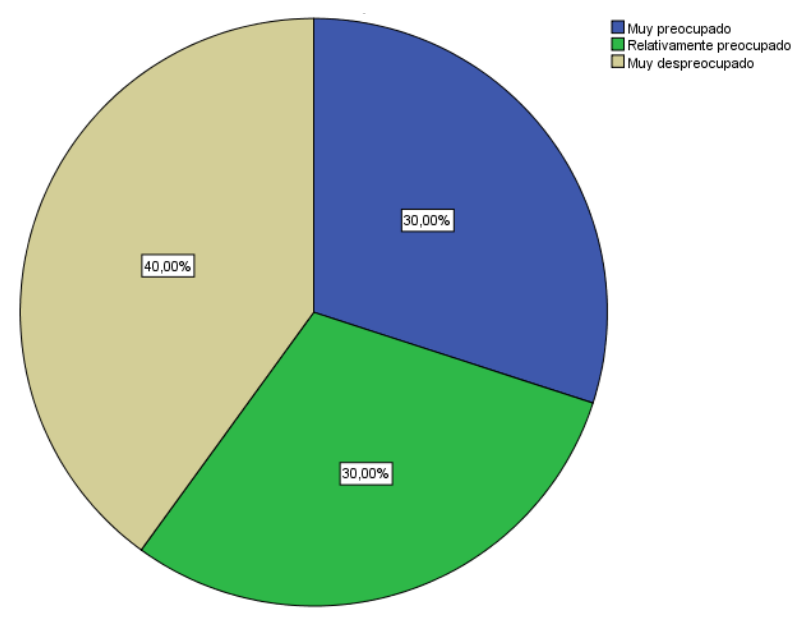

\section{Figura 4.}

Trabajadores de las empresas públicas y empresas privadas con preocupación por la conservación y mejora del medio ambiente. Fuente: Elaboración propia.

\section{Discusión}

Este trabajo de investigación tuvo como objetivo determinar cómo la gestión ambiental de las empresas públicas y privadas incide en el desarrollo sostenible, aplicado en el cantón Guayaquil.

La principal aportación de este estudio es que, a diferencia de otras investigaciones permite identificar si la gestión ambiental de las empresas públicas y privadas es la adecuada. El enfoque de esta investigación se basa en un diseño exploratorio mediante la aplicación de un cuestionario realizado a las diferentes empresas que permite indagar sobre su gestión ambiental y la forma en que inciden en el desarrollo sostenible.

La mayoría de las investigaciones han evaluado la gestión de las empresas por separado y no se ha hecho un estudio que involucre el sector gubernamental y no gubernamental. Estos resultados tienen diversas implicaciones para la comunidad académica, empresarial y gubernamental. Para los investigadores estos resultados abonan el conocimiento sobre gestión ambiental desde una perspectiva poco 
analizada que es la del sector público y privado, que permite conocer la gestión ambiental desde distintas ópticas.

Así mismo, los resultados confirman que la gestión ambiental de las empresas públicas y privadas no son determinantes para el desarrollo sostenible, de acuerdo a lo que se describe a continuación:

Rodríguez-Córdova (2016) define a la gestión ambiental de las organizaciones como el conjunto de las acciones dirigidas a la administración y correcto uso de los recursos naturales para su conservación controlando las actividades realizadas por las personas, lo cual resulta coincidente con la investigación porque los resultados muestran que el 50\% de las empresas entrevistadas aplican las Buenas Prácticas Ambientales de Energía, agua, desechos, papel y responsable consumo.

De igual manera, Rodríguez-Córdova (2016) considera que la gestión ambiental necesita la aplicación de políticas ambientales que involucren la cultura de los individuos, experiencias y participación ciudadana. Por lo tanto, se encuentra coincidencia con la investigación porque los resultados describen que el $80 \%$ de las empresas del estudio han implementado políticas ambientales.

Se puede apreciar que existe relación con lo manifestado por Hernández (2015), quien concluyó que "El estado es el encargado de formar un talento humano responsable con la inclusión de temas ambientales en los diferentes programas de estudio y disciplinas para obtener el desarrollo sostenible", y los resultados muestran que el $70 \%$ de las instituciones de la investigación tienen personal capacitado en temas ambientales que respaldan esta afirmación.

Finalmente, Severiche (2016) plantea que se debe fortalecer la educación y mejorar la conciencia de todos los ciudadanos fomentando el respeto a todo lo que tenga vida, por lo cual, se evidencia una contradicción con los resultados del estudio debido a que el $40 \%$ de los trabajadores de las empresas públicas y privadas no tienen ninguna preocupación por el daño ambiental causado al planeta debido a que la formación de los individuos no ha sido suficiente para crear conciencia por el medio que nos rodea. 
La principal limitación de la investigación es que los resultados no pueden generalizarse pues en la muestra se han utilizado empresas que se dedican a diferentes actividades. Por lo anterior, futuras investigaciones deben centrarse en realizar estudios en otras empresas que se dediquen a la misma actividad económica, pero con el enfoque de la perspectiva de la gestión ambiental y aplicación de Buenas Prácticas Ambientales, para estar en posición de generalizar los resultados encontrados e ir incorporando nuevas variables de análisis en la gestión ambiental. Así mismo, cabría realizar el estudio en distintas regiones geográficas del país para estar en posición de realizar estudios comparativos y de esta manera, profundizar el análisis de las causas por las cuales la sostenibilidad enfrenta dificultades a la hora de convertirse en prácticas concretas.

\section{Conclusiones}

Los resultados obtenidos en la recopilación de datos demostraron que la gestión ambiental de las empresas públicas y privadas no son determinantes para el desarrollo sostenible. En lo que respecta a las Buenas Prácticas Ambientales, se presentó el 50\% de empresas aplican la (Energía, agua, desechos, papel y responsable consumo). E1 70\% de los entrevistados se encuentran (Totalmente de acuerdo) en que las acciones realizadas en su organización inciden en el desarrollo sostenible. Así mismo, cuentan con el 70\% de personal capacitado en temas ambientales y el $40 \%$ de los trabajadores de las empresas públicas y privadas se sienten muy despreocupados por la conservación y mejora del medio ambiente, por lo que los resultados confirman que la gestión ambiental de las empresas públicas y privadas no son determinantes para el desarrollo sostenible.

En la actualidad, las empresas deben adaptarse al entorno, por lo que deben reinventarse continuamente para responder a los cambios y a las nuevas exigencias que los tiempos demandan. Por otra parte, las instituciones gubernamentales deben reconocer y recompensar a las empresas que realizan acciones de sostenibilidad, y a su vez, es necesario que las organizaciones y los gobiernos trabajen en conjunto para diseñar políticas y generar beneficios que impulsen a las organizaciones a conseguir mejores resultados de sostenibilidad. 
Adicionalmente, muchas empresas tienen dificultades para reconocer las ventajas que representa el ser sostenibles, por lo que también se debe considerar brindarle orientación al respecto. Se debe tener en cuenta que todo proceso de transformación lleva tiempo, especialmente cuando se trata de cambiar formas de pensamiento y creencias arraigadas.

El desarrollo sostenible empresarial solo se alcanzará cuando las organizaciones trabajen por la ecoeficiencia; para ello las empresas deben implantar un conjunto de herramientas de gestión ambiental organizacional y cumplir con las regulaciones ambientales aplicables vigentes.

\section{Referencias}

Bermejo, R. (2014). Del desarrollo sostenible según Brundtland a la sostenibilidad como biomimesis. Recuperado de http://publicaciones.hegoa.ehu.es/uploads/pdfs/253/Sostenibilidad_DHL. pdf?1488539808

Blázquez, M., y Peretti, M. (2012). Modelo para gestionar la sustentabilidad de las organizaciones a través de la rentabilidad, adaptabilidad e imagen. Estudios Gerenciales, 28, 40-50, doi: 10.1016/S0123-5923(12)70006-2.

Carro, J., Sarmiento, S., y Rosano, G. (2017). La cultura organizacional y su influencia en la sustentabilidad empresarial. La importancia de la cultura en la sustentabilidad empresarial. Estudios Gerenciales, 33, 352-365, doi: 10.1016/j.estger.2017.11.006

Chirinos, M., Fernández, L., y Sánchez, G. (2012). Responsabilidad empresarial o empresas socialmente responsables. Razón y Palabra, 81, 118. Recuperado de http://www.redalyc.org/articulo.oa?id=199524700002

Climent, V., y Giner, S. (2001). Empresa y problemática ambiental. Un análisis sociológico. Revista Internacional de Sociología, 59(29), 115132, doi: 10.3989/ris. 2001.i29.758

Gómez, J. (2014). Del desarrollo sostenible a la sustentabilidad ambiental. Investigación y Reflexión, 22(1), 115-136. Recuperado de https://www.redalyc.org/pdf/909/90931814009.pdf 
Hernández, E. (2015). Ambiente, gestión ambiental. Avances y retrocesos del ambiente y desarrollo sustentable en Venezuela. Provincia Universidad de Los Andes, 34, 97-116.

Hernández, M. A., Mendoza, J., y Salazar, B. C. (2017). La responsabilidad social en la empresa familiar. Holos, 5, 174-185, doi: 10.15628/holos.2016.4805.

Isaac, C., Gómez, J., y Díaz, S. (2017). La integración de herramientas de gestión ambiental como práctica sostenible en las organizaciones. Universidad y Sociedad, 9(4), 27-36. Recuperado de http://scielo.sld.cu/scielo.php?script=sci_arttext\&pid=S221836202017000400004

López, A., Ojeda, J., y Ríos, M. (2017). La responsabilidad social empresarial desde la percepción del capital humano. Estudio de un caso. Revista de Contabilidad, 20(1), 36-46, doi: 10.1016/j.rcsar.2016.01.001.

Ministerio del Ambiente del Ecuador. (2010). Acuerdo Ministerial 131Políticas generales para promover las buenas prácticas ambientales en entidades del sector público. MAE. Recuperado de http://www.ambiente.gob.ec/wpcontent/uploads/downloads/2012/10/Acuerdo-Ministerial-131.pdf

ONU. (2015). Transformando nuestro mundo: la Agenda 2030 para el Desarrollo Sostenible. Cumbre del Milenio. Recuperado de https://www.ar.undp.org/content/dam/argentina/Publications/Agenda2030 /PNUDArgent-DossierODS.pdf

Riestra, L. (2018). Las Dimensiones del Desarrollo Sostenible como Paradigma para la Construcción de las Políticas Públicas en Venezuela. Rev. Tekhné, 2l(1), 24-33. Recuperado de http://revistasenlinea.saber.ucab.edu.ve/temas/index.php/tekhne/article/vi ew/3543/3041

Río+20. (2012). El futuro que queremos. Recuperado de http://www.oitcinterfor.org/sites/default/files/file_evento/docfinalrio20.p $\mathrm{df}$

Rodríguez-Córdova, R. (2016). Fundamentos básicos para la ejecución de la auditoría ambiental. Ciencias Holguín, 22(1), 1-18. Recuperado de http://www.redalyc.org/articulo.oa?id=181543577002

Severiche, C., Gómez, E., y Jaimes, J. (2016). La educación ambiental como base cultural y estrategia para el desarrollo sostenible. Telos. Revista de Estudios Interdisciplinarios en ciencias sociales., 18(2), 266-281. Recuperado de http://www.redalyc.org/articulo.oa?id=99345727007 
WBCSD. (2000). Eco-efficiency: Doing more with less. Recuperado de http://libroweb.alfaomega.com.mx/book/733/free/ovas_statics/unid2/PDF _Ingles/Eco_Efficiency.pdf

Zarta, P. (2018). La sustentabilidad o sostenibilidad: un concepto poderoso para la humanidad. Tabula Rasa, 28, 409-423, doi: 10.25058/20112742.n28.18. 